\title{
What Advice Can We Give to Optimize Our Patients' Odds of Aging with Better Health?
}

\author{
Rita Manuela Vieira Gomes ${ }^{1}$, Oliveira S. ${ }^{2}$, Pires S. ${ }^{3}$, Alves J. ${ }^{1}$ and Silva R. ${ }^{1}$ \\ 1. S. João de Braga Family Health Unit, 4710-488 Braga, Portugal \\ 2. Family Health Unit of Minho, Portugal \\ 3. Family Health Unit of Régua, Portugal
}

\begin{abstract}
The world's population is ageing rapidly, which will have substantial consequences. According to WHO: "Process of optimizing opportunities for health, participation and security in order to enhance quality of life as people age". The aim of this work is to make a literature review on key measures that contribute to healthy ageing. A search was conducted in the following databases: PubMed, Cochrane, National Guideline Clearinghouse, Trip Database, Guideline finders, NICE and WHO database, written in English and published in the last ten years. The following MeSH words were used: "healthy ageing". The search was conducted from July first till end of December 2015. Based on the articles searched there are many measures with potential to achieve gains in healthy ageing such as prevention of falls; vaccination; regular physical activity, eating fruits and vegetables daily, drinking alcohol moderately; never smoking; prevention of social isolation and social exclusion; prevention of elder maltreatment; public support for informal care giving and create strategies to ensure the quality of care for older people. Getting older can come with a variety of health challenges. In the daily practice of family doctor there are many effective interventions that can improve health and wellbeing in elderly people.
\end{abstract}

Key words: Healthy ageing, elderly, active ageing.

\section{Introduction}

The continue increases in life expectancy make remaining free of disease and in good functional health for as long as possible an important objective for the present and future generations [1].

The world's population is aging rapidly, the consequences of which will be substantial.This trend risks overwhelming health and social support systems with millions of older adults, many of whom will have chronic pain, serious age related diseases and disability [2]. So, in a growing elderly population, healthy ageing is becoming a crucial factor to reduce the burden of disease and disability and related healthcare costs [3].

"Healthy Ageing" is a short term for the broader concept of both active and healthy ageing,it is defined

Corresponding author: Rita Manuela Vieira Gomes, research field: primary care. by the World Health Organization (WHO) as the "process of optimizing opportunities for health, participation and security in order to enhance quality of life as people age. Active ageing allows people to realize their potential for physical, social, and mental well-being throughout the life course and to participate in society according to their needs, desires and capacities, while providing them with adequate protection, security and care when they require assistance" [4, 5].

However, despite the best efforts in health promotion and disease prevention, people are at increasing risk of developing diseases as they grow older [5].

In this context, one of the key targets recently outlined by the European Commission's Active and Healthy Ageing Innovation Partnership is to achieve an increased healthy life expectancy of 2 years by the year 2020 [6]. Healthy life expectancy is commonly used as a synonym for "disability-free life 
expectancy". While life expectancy at birth remains an important measure of population ageing, how long people can expect to live without disabilities is especially important to an ageing population [5].

In light of the above, the aim of this work is to make a literature review on key measures that contribute to healthy and active ageing.

\section{Methods}

A search was conducted in the following databases: PubMed, Cochrane, National Guideline Clearinghouse, Trip Database, Guideline finders, National Institute for Health and Clinical Excellence (NICE) and WHO database. We restricted this search only to articles published in Englishin the last ten years.

The following MeSH words were used: "healthy ageing". The search was conducted from July first till end of December 2015.

\section{Results}

From the analysis and review of all the articles found we have concluded that there are five essential measures that all physicians should recommend and defend for their patients in order to help them to have a healthier and more active ageing.

As such, the main measures were grouped into five categorieswhich are: regular physical activity, prevention of falls, healthy diet, mental well-being and active ageing policies.

\subsection{Regular Physical Activity}

Physical activity is a modifiable behaviour associated with health, functional status, and longevity, and encouraging a physically active lifestyle has become an accepted public health goal [7].

In fact, physical activity has been shown to have beneficial effects both on physical and cognitive function in older people and has been associated with increased survival in the elderly [8-10].

Moreover, sustained physical activity was prospectively associated with improved healthy ageing, such as absence of disease, freedom from disability, high cognitive and physical functioning and good mental health. Significant health benefits were even seen among people who became physically active relatively late in life [11].

NICE guidelines recommend that older people should be offered tailored exercise and physicalactivity programmes in the community, focusing on: a range of mixed exercise programmes of moderate intensity (for example, dancing, walking, swimming); strength and resistance exercise, especially for frail older people and toning and stretching exercise. Older people should be encouraged to attend sessions at least once or twice a week.Notwithstanding, it is also important that we ensure that exercise programmes reflect the preferences of older people [12].

NICE also recommend to advise older people and their carers how to exercise safely for 30 minutes a day (which can be broken down into 10-minute bursts) on 5 days each week or more. In addition, these guidelines provide useful examples of activities in daily life that would help achieve this, such as shopping, housework, gardening and cycling [12].

\subsection{Prevention of Falls}

Falls among older people and the injuries to which they often lead are the underlyingcauses of a large share of the burden of disease and disability among older people in Europe and a major risk factor for developing frailty. The risk of falls increases steeply with age. Injuries from falls (such as femur fracture) usually require hospitalization and costly interventions, including rehabilitation. They are the underlying cause of many of the functional limitations that lead to the need for long-term care, including admission to a nursing home. Environmental hazards account for between a quarter and a half of falls; other factors include muscle weakness, gait and balance disturbances, previous history of falls, and medication. Falls can happen in any setting: $30-40 \%$ of nursing 
home residents have been reported to fall each year. There is convincing evidence that most falls are preventable [13].

To prevent falls, a multifactorial approach is necessary.In successful multifactorial intervention programmes the following specific components are common: strength and balance training; home hazard assessment and intervention; vision assessment and referral and medication review with modification/withdrawal. Following treatment for an injurious fall, older people should be offered a multidisciplinary assessment to identify and address future risk and individualised intervention aimed at promoting independence and improving physical and psychological function [14].

So, healthy feet are essential for safe walking and to maintain mobility. It's essential to remember the elderly to wash their feet daily and to keep toenails short. Choosing the best footwear may improve balance and stability. Discard any eye and hear conditions that develop with aging. Alert to some home conditions like: advice to use well-fitting slippers, replace loose rugs or frayed carpets, make sure to have good lighting, especially on stairs. It's also essential not to forget review medicines regularly, taking into account, mainly the elderly who take psychotropic medications [13-15].

\subsection{Healthy Diet}

Eating and food security problems at all ages include both under-nutrition (mostly, but not exclusively, in the least developed countries) and excess energy intake. In older people, malnutrition can be caused by limited access to food, socioeconomic hardships, a lack of information and knowledge about nutrition, poor food choices, disease and the use of medications, tooth loss, social isolation, cognitive or physical disabilities that inhibit one's ability to buy foods and prepare them, emergency situations and a lack of physical activity. Excess energy intake greatly increases the risk for obesity, chronic diseases and disabilities as people grow older [5].

Furthermore, insufficient calcium and vitamin D is associated with a loss of bone density in older age and consequently an increase in painful, costly and debilitating bone fractures, especially in older women. In populations with high fracture incidence, risk can be decreased through ensuring adequate calcium and vitamin D intake [5].

On the other side, moderate alcohol consumption and not smoking have well-known health benefits, so it is notsurprising that they may affect odds for successful aging. Similarly, although the number of fruits and vegetables to be eaten daily was not quantified, consumption of at least some amounteach day appears to have an important association with successful aging [16].

\subsection{Mental WellBeing}

According to WHO "mental health is defined as a state of well-being in which every individual realizes his or her own potential, can cope with the normal stresses of life, can work productively and fruitfully, and is able to make a contribution to her or his community".

NICE guidelines recommend occupational therapy interventions, where a regular group and/or individual sessions to encourage older people to identify, construct, rehearse and carry out daily routines and activities that help to maintain or improve their health and wellbeing. This sessions should: involve older people as experts and partners in maintaining or improving their quality of life; pay particular attention to communication, physical access, length of session and informality to encourage the exchange of ideas and foster peer support; take place in a setting and style that best meet the needs of the older person or group and they will provide practical solutions to problem areas [12].

Also, in thisoccupational therapy it is necessary increase older people's knowledge and awareness of where to get reliable information and advice on a 
broad range of topics, by providing information directly, inviting local advisers to give informal talks, or arranging trips and social activities. Topics covered should include:meeting or maintaining healthcare needs; nutrition; personal care; staying active and increasing daily mobility; getting information on accessing services and benefits; home and community safety and using local transport schemes [12].

In addition, it is important that in all medical consultations depression screening is carried out because it is a relatively frequent condition in the elderly and subdiagnosed, as well as to rule out bladder control problems, whichis a frequent condition too in the elderly, yet many people keep it a secret for years, bringing them a lot of suffering and disability $[5,15]$.

It is also known that the practice of physical exercise improves mental health [11].

\subsection{Active Ageing Policies}

An active ageing approach to policy and programme development has the potential to address many of the challenges of both individual and population ageing. When health, labour market, employment, education and social policies support active ageing there will potentially be: fewer premature deaths in the highly productive; stages of life; fewer disabilities associated with chronic diseases in older age; more people enjoying a positive quality of life as they grow older; more people participating actively as they age in the social, cultural, economic andpolitical aspects of society, in paid and unpaid roles and in domestic, family and community life; lower costs related to medical treatment and care services [5]. Considering the above we have concluded that it is essential topromote age-friendly policies and environments, such as, forexample, physical environments that are age friendly can make the difference between independence and dependence for all individuals but are of particular importance for those growing older $[5,15]$.
Additionally, older people who live in an unsafe environment or areas with multiple physical barriers are less likely to get out and therefore more prone to isolation, depression, reduced fitness and increased mobility problems [5].

Another important measurement is also to establish good programmes of vaccination, specially, for Flu and Pneumococcal vaccination $[5,15,17]$.

NICE guidelines recommend support, publicise and, if there is not enough provision, consider providing a range of group, one-to-one and volunteering activities that meet the needs and interests of local older people. In particular, target older people who are identified as being most at risk of a decline in their independence and mental wellbeing. For example, older people whose partner has died in the past 2 years are at risk. Others at risk includes those who: are carers; live alone and have little opportunity to socialise; have recently separated or divorced; have recently retired (particularly if involuntary); were unemployed in later life; have a low income; have recently experienced or developed a health problem (whether or not it led to admission to hospital); have had to give up driving; have an age-related disability and are aged 80 or older [18].

Moreover, NICE also recommends to involve older people in the design and delivery of activities. For this purpose it is necessary, namely, to ensure that each activity: includes a clear description of what is on offer; takes place at regular times and in a regular location; provides the opportunity to socialise; complements other activities that may support different aspects of older people's independence and mental wellbeing, such as their physical health, their sense of belonging to a community ('social connectedness') and their sense of purpose. In addition, it should also be ensured that the activities are inclusive and take account of a range of different needs [18].

Although individual healthy behaviours are moderately associated with successful aging, their 
combined impact is quite substantial. In fact, multiple healthy behaviours appear to increase the chance of reaching old age disease-free and fully functional in an additive manner [16].

\section{Conclusions}

Getting older comes with a variety of health challenges. In the daily practice of a family doctor there are many effective interventions that can improve the health and well-being of elderly people. Professional caregivers need training and practice in enabling models of care that recognize older people's strengths and empower them to maintain even small measures of independence.

Global ageing does require governments and the private sector to address the challenges on social protection and to create age-friendly environments. Also, these programmes should encourage inactive people to become more active as they age and to provide them opportunities to do so. An active ageing approach seeks to eliminate age discrimination and recognize the diversity of older populations. Older people and their caregivers need to be actively involved in the planning, implementation and evaluation of policies, programmes and knowledge development activities related to active ageing.

The research on human aging has a bright future and its results have the potential to continue influencing clinical decision-making and healthy policy.

In this context, it is time for a new paradigm, one that views older people as active participants in an age-integrated society and as active contributors as well as beneficiaries of development. Finally, genomic, epigenomic, proteomic and metabolomic studies are just beginning to assessthe genetic and environmental components of healthy aging with systems approaches, which will ultimately help us better understand the complex relations that determine the odds of healthy aging. Considering the diversity of fresh initiatives and different approaches there is a greater hope that we are on the verge of findings that will lead to a much healthier old age.

\section{References}

[1] Christensen, K., Doblhammer, G., Rau, R., et al. 2009. "Ageing populations: the challenges ahead." Lancet 374: 1196-208.

[2] Sinha, S. K. 2011. "Why the elderly could bankrupt Canada and how demographic imperatives will force the redesign of acute care service delivery." Healthc Pap 11: 46-51.

[3] Landefeld, C. S., Winker, M. A., Chernof, B. 2009. "Clinical care in the aging century-announcing "Care of the aging patient: from evidence to action'.” JAMA 302: 2703-4.

[4] World Health Organization, Active Ageing, Good health adds life to years, Policies and priority interventions for healthy ageing, 2012.

[5] World Health Organization, Active Ageing: A Policy Framework, 2002.

[6] Lagiewka, K. 2012. "European innovation partnership on active and healthy ageing: triggers of setting the headline target of 2 additional healthy life years at birth at EU average by 2020." Arch Public Health 70: 23.

[7] Haskell, W. L., Lee, I. M., Pate, R. R., et al. 2007. "American College of Sports Medicine; American Heart Association, Physical activity and public health: updated recommendation for adults from the American College of Sports Medicine and the American Heart Association." Circulation 116 (9): 1081-93 PubMed.

[8] Villareal, et al. 2011. "Weight Loss, Exercise, or Both and Physical Function in Obese Older Adults." N. Engl. J. Med. 364: 1218-29.

[9] Lautenschlager, N. T., Cox, K. L., Flicker, L., et al. 2008. "Effect of Physical Activity on Cognitive Function in Older Adults at Risk for Alzheimer Disease A Randomized Trial.” JAMA 300 (9): 1027-37. doi:10.1001/jama.300.9.1027.

[10] Stessman, J., Ginsberg, G., Klein, M., Hammerman-Rozenberg, R., Friedman, R., Cohen, A., et al. 2009. "Physical Activity, Function, and Longevity among the Very Old." Arch Intern Med. 169 (16): 1476-83. doi:10.1001/archinternmed.2009.248.

[11] Hamer, M., Lavoie, K. L., and Bacon, S. L. 2014. "Taking up physical activity later in life and healthy ageing: the English longitudinal study of ageing." $B r . J$. Sports Med. 48: 239-43.

[12] National Institute for Health and Clinical Excellence (NICE) (2008), Mental wellbeing in over 65s: occupational therapy and physical activity interventions. Available at:www.nice.org.uk/guidance/ph16/chapter/1-Recommen 
dations.[Accessed: 26 November 2015].

[13] World Health Organization, Global Report on Falls Prevention in Older Age. Geneva, 2007. Available at:ww.who.int/entity/ageing/publications/Falls_preventio n7March.pdf. [Accessed: 26 November 2015].

[14] National Institute for Health and Clinical Excellence (NICE) (2013), Falls in older people: assessing risk and prevention. Available at: www.nice.org.uk/guidance/ cg161/chapter/1-Recommendations. [Accessed: 27 November 2015].

[15] NHS England, Age UK, Practical Guide to Healthy Ageing. [Accessed: 27 November 2015].

[16] Sabia, S., Singh-Manoux, A., Hagger-Johnson, G., et al.
2012. "Influence of individual and combined healthy behaviours on successful aging." CMAJ 184: 1985-92.

[17] World Health Organization Regional Office for Europe, Policies and priority interventions for healthy ageing, 2012. Available at: //www.euro.who.int/_data/assets/ pdf_file/0006/161637/WHD-Policies-and-Priority-Interve ntions-for-Healthy-Ageing.pdf?ua $=1 \quad$ [Accessed: 8 November 2015].

[18] National Institute for Health and Clinical Excellence (NICE) (2013), Older people: independence and mental wellbeing. Available at: www.nice.org.uk/guidance/ng32/chapter/ Recommendations. [Accessed: 27 November 2015]. 